\title{
Didactique(s) : quel dialogue au sein des sciences de l'homme et de la société ? Une introduction
}

Didactics and Social Sciences interactions: an introduction

Corinne Marlot et Jean-Charles Chabanne

\section{OpenEdition}

\section{Journals}

\section{Édition électronique}

URL : https://journals.openedition.org/educationdidactique/2546

DOI : 10.4000/educationdidactique. 2546

ISSN : $2111-4838$

Éditeur

Presses universitaires de Rennes

\section{Édition imprimée}

Date de publication : 19 décembre 2016

Pagination : $9-19$

ISBN : 978-2-7535-5372-9

ISSN : 1956-3485

Référence électronique

Corinne Marlot et Jean-Charles Chabanne, « Didactique(s) : quel dialogue au sein des sciences de I'homme et de la société ? Une introduction », Éducation et didactique [En ligne], 10-3 | 2016, mis en ligne le 19 novembre 2018, consulté le 22 juillet 2022. URL : http://journals.openedition.org/ educationdidactique/2546; DOI : https://doi.org/10.4000/educationdidactique.2546 


\title{
DIDACTIQUE(S) : QUEL DIALOGUE AU SEIN DES SCIENCES DE L'HOMME ET DE LA SOCIÉTÉ ? UNE INTRODUCTION
}

\author{
Corinne Marlot \\ HEP Vaud, UER MS (CH) et laboratoire ACTÉ, université Blaise-Pascal, Clermont-Ferrand (FR) \\ Jean-Charles Chabanne \\ ENS de Lyon, IFÉ-UMS 3773 et laboratoire ACTÉ, université Blaise-Pascal, Clermont-Ferrand (FR)
}

\begin{abstract}
Un(e) didacticien(ne) est à la fois un(e) ingénieur(e) et un(e) chercheur(e) en didactique qui intervient dans des situations et sur des objets qui relèvent d'approches larges et complexes de la « culture » et du « social ». Il / elle est et non seulement concepteur de dispositifs et de processus d'enseignement : mais il / elle doit aussi prendre en compte l'activité des individus à l'intérieur et à l'extérieur des institutions d'enseignement, d'un point de vue multicontextuel. Les multiples références aux environnements humains, sociétaux, politiques et éthiques, où se déroulent les phénomènes d'enseignement-apprentissage observés, élargissent nécessairement le champ d'intervention du didacticien. Les constructions didactiques théoriques et méthodologiques impliquent des relations complexes avec les Sciences Humaines et Sociales, en relation avec les différentes questions traitées par les approches didactiques. Le présent numéro donne à voir les travaux du deuxième atelier scientifique co-organisé par l'association ARCD et la revue Éducation \& Didactique, dans la perspective de problématiser la géométrie et les enjeux de la didactique en questionnant leurs positionnements à l'intérieur des Sciences Humaines et Sociales.
\end{abstract}

Mots-clés : didactique comparée, didactique(s) et sciences sociales.

\section{Didactics and Social Sciences interactions: an introduction}

A scholar in didactics/instruction design is both an engineer and a researcher working on situations and objects, using broad and complex "cultural" and "social" approaches. He/she is not only a designer of instruction devices and processes but hel she also has to take into account individual's activities inside and outside educational institutions, from a multicontextual point of view. Observed teaching-learning phenomena having multiple references to the human, societal, political and ethical contexts, the searcher field of intervention is undoubtedly wide. The theoretical and methodological didactic constructions involve complex relationships with the Social Sciences, to treat the questions raised by the different didactic approaches. This issue exposes the results produced at the second scientific workshop co-organized by the association ARCD and the journal Education \& Didactique, in order to problematize the geometry and purposes of different didactics, while questioning their status within Human and Social Sciences.

Keywords: comparative didactics/instruction design, didactics/instruction design and social sciences 
Dans l'univers francophone des recherches en éducation, depuis plus d'une trentaine d'années, les didactiques ont connu un essor important dans le contexte de la massification de l'enseignement secondaire et des grandes réformes du curriculum qui ont accompagné cette transformation de l'École. Dès les années quatre-vingt-dix, le tournant de la professionnalisation du métier d'enseignant, allant de pair avec une universitarisation croissante de la formation, a vu se développer de nombreux programmes de recherche centrés sur le travail de l'enseignant et ses déterminants (épistémologie pratique, rapport aux savoirs...), mais aussi sur l'analyse des pratiques en classe ordinaire et les conditions de leur transformation, au travers de la mise en place de processus de recherche collaboratifs, faisant évoluer la notion d'ingénierie didactique. Par ailleurs, une préoccupation constante reste la réduction des inégalités d'accès aux savoirs dans les systèmes scolaires avec la nécessité de prendre en compte les difficultés des élèves, le problème de la différenciation, etc.

Les questions liées aux contenus disciplinaires n'ont pas disparu pour autant : elles évoluent, sous la pression institutionnelle qui demande, d'une part, que soit défini un socle de savoirs fondamentaux au sein desquels les savoirs langagiers tiennent une place particulière, d'autre part, que de nouvelles formes d'apprentissage permettent au futur citoyen de développer son autonomie et sa créativité, notamment à travers les "éducations à", les parcours d'étude et de recherche, les Enseignements Pratiques Interdisciplinaires, et autres démarches fondées sur l'investigation. Le questionnement et l'analyse des contenus enseignés-appris à l'École reste une composante identitaire forte dans les recherches en didactique (voir par exemple Daunay, Fluckiger \& Hassan, 2015).

Ces différentes problématiques (et sans doute bien d'autres que nous ne pouvons évoquer ici) ont contribué au besoin d'étendre les recherches didactiques hors de leurs domaines disciplinaires d'origine, pour se situer désormais à la croisée de certains d'entre eux, afin de saisir des objets aux frontières d'une ou plusieurs disciplines, tout en cherchant à tenter une approche systémique du tripôle enseignant-élève-savoir. Il en résulte un champ de recherches traversé par des relations complexes qui justifient tantôt de parler d'une pluralité de "didactiques" et tantôt de parler d'une "didactique" au singulier ; relations que nous avons tenté de décrire et de problématiser lors de la précédente journée organisée conjointement par l'ARCD (Association pour de Recherches Comparatistes en Didactique) et la revue Éducation \& Didactique (voir son numéro 8, volume $1^{1}$ ). Les évolutions curriculaires actuelles et les enjeux de formation afférents, aussi bien que les nouveaux objets et dispositifs qui apparaissent dans l'arène scolaire, renouvellent les questions posées aux didacticiens : " autant de questions qui semblent difficiles à traiter depuis les seuls cadres épistémologiques de quelques disciplines bien délimitées * (Ligozat et al., 2014).

La deuxième journée ARCD et Éducation $\mathcal{E}$ Didactique, qui s'est tenue à l'ENS de Lyon le 11 novembre 2015, a poursuivi le travail de problématisation des contours et des enjeux de ce champ de recherches, en questionnant son positionnement dans et en relation avec les autres sciences de l'homme et de la société. Une telle question est évidemment très vaste, et cette seule journée ne saurait couvrir l'ensemble des champs connexes. Le travail engagé est à long terme.

D'une part, depuis sa création en 2005, l'ARCD 2 s'est donné pour but d'« inscrire les problématiques comparatistes, relatives aux conditions individuelles, institutionnelles et sociales de la transmission et du partage des savoirs et des pratiques, dans le champ des SHS » (Statuts de l'ARCD). D'autre part, la revue Éducation \& Didactique propose de « repenser les rapports entre les didactiques à l'intérieur des recherches en éducation et de développer les relations entre la didactique et les autres sciences de l'homme et de la société » (voir le site de la revue ${ }^{3}$ ). Le débat engagé lors de la journée de 2015 nous amène à réfléchir selon deux axes.

\section{UN AXE ÉPISTÉMOLOGIQUE}

Il paraît difficile d'imaginer une "science du didactique »-pour reprendre un terme posé au cœur du projet de la didactique comparée par Mercier, Schubauer-Leoni et Sensevy (2002) - qui étudie les conditions de diffusion et de transmission des savoirs dans la société, sans une réflexion approfondie sur les pratiques de production des savoirs dans différentes sphères sociales, d'une part, et d'autre part, sur l'activité des acteurs qui contribuent à la diffusion / transmission. Le didacticien est à la fois un ingénieur et un chercheur qui intervient dans des situations 
et sur des objets qui relèvent d'approches larges et complexes de la "culture" et du "social" et pas seulement un concepteur de situations d'enseignement et d'apprentissage au service d'une institution donnée. En conséquence, le didacticien est amené à prendre en compte l'activité des sujets en prise avec des institutions scolaires et non scolaires. Les multiples références aux contextes humains, sociétaux, politiques, éthiques dans lesquels s'inscrivent les phénomènes d'enseignement et d'apprentissage observés élargissent nécessairement le domaine d'intervention du didacticien. Les constructions théoriques et méthodologiques des didacticiens entretiennent des rapports complexes aux SHS, en lien avec les différentes questions que traitent les approches didactiques (pour exemple, Bronckart, 1987 ou plus tard, Bernié, 2002). Quels sont alors les emprunts effectués par rapport à d'autres disciplines des SHS ? Quels sont les concepts empruntés, et selon quelles adaptations ? En quoi cela affecte-t-il rapports établis avec les sciences dites "de référence », qui contribuent à assurer une légitimité aux savoirs enseignés à l'École?

Dans l'autre sens, quels sont, par exemple, les textes fondamentaux du champ des / de la didactique(s) qui sont cités dans les autres domaines ? Ou encore, quel est le degré de connaissance et de diffusion, dans les sciences sociales, des questionnements, des méthodes et éventuellement des concepts de la didactique ou des didactiques? Les représentations dépréciatives de la / des didactique(s) comme relevant de l'ingénierie, et, dans les analyses, la tension entre sciences comme connaissance et sciences comme pratique (éventuellement transformatrice) peuvent-elles expliquer en partie les difficultés à dialoguer parfois avec les autres disciplines des SHS?

\section{UN AXE OPÉRATOIRE}

Les nombreuses interactions entre didacticiens et sociologues de l'éducation, par exemple, rendent compte à la fois de problématiques partagées mais aussi de différences de traitement de ces problématiques. Nous ne pouvons ignorer le long dialogue qui eut lieu en janvier 1998 à l'université d'Aix Marseille entre Samuel Johsua et Bernard Lahire, en présence d'étudiants et de collègues appartenant à différentes disciplines des sciences humaines et sociales (Johsua
$\&$ Lahire, 19994). Ce dialogue avait pour but d'envisager les obstacles et les possibilités d'un rapprochement de la didactique avec la sociologie dans les années à venir. Ce rapprochement est balisé par la suite par certaines publications importantes telles que La sociologie, la didactique et leurs domaines scientifiques (Lahire, $2007^{5}$ ), les actes du colloque de Lausanne Sociologie et didactique : vers une transgression des frontières en 2012 (Losego, 2014a ${ }^{6}$ ), le numéro 188 de la Revue française de pédagogie (Losego, 2014b $\mathrm{b}^{7}$ ), le numéro 8 , volume $1^{8}$ de la revue Éducation \& Didactique. La réflexion se poursuit ici.

Comment réduire l'échec scolaire en prenant en compte ce qu'en dit la sociologie des apprentissages (Rayou, 2014) ? Comment permettre au plus grand nombre d'accéder à une scolarité désormais prolongée ? Comment assurer la réussite pour tous d'un apprentissage "tout au long de la vie " ? Ce sont des questions éminemment complexes qui, de notre point de vue, rendent le rapprochement nécessaire. En quoi la collaboration avec certaines disciplines des SHS (sociologie et histoire de l'éducation, philosophie de l'éducation, sciences du langage, sciences de l'information et de la communication, etc.), permet-elle de mieux comprendre les effets des choix éducatifs et scolaires de nos sociétés? À quelles conditions ce rapprochement est-il fécond ? L'existence de lieux institutionnels comme RESEIDA (Regroupement pour des Recherches sur la Socialisation, l'Enseignement, les Inégalités et les Différenciations dans les Apprentissages) ou encore l'IFÉ (Institut français de l'Éducation), au sein desquels la question des liens entre didactique(s) et autres SHS est posée, contribuent à créer des conditions de collaboration fructueuses (Rochex \& Crinon, 2011 ; Laparra \& Margolinas, 2016). Dès lors, comment se modèlent les lignes de partage des problématiques, des concepts, des méthodes, quand des didacticiens travaillent conjointement avec des chercheurs de ces disciplines? En quoi, par exemple, l'analyse de corpus communs et la rencontre de concepts issus de champs différents entrant alors en résonance et en tension, permettent-elles une co-problématisation plus dense ? En quoi les percolations qui en découlent ouvrent-elles sur une meilleure compréhension de la façon dont s'élaborent les logiques sociales des différents types d'acteur à l'École ? Comment les didacticiens peuvent-ils échanger sur les méthodes de production et de traitement des observables avec d'autres domaines des 
SHS ? Les méthodologies utilisées dans l'un ou l'autre des champs sont-elles spécifiques ou transférables à l'autre? Sous quelles conditions ou conversion ? Existe-t-il un espace permettant de faire connaitre et mettre en discussion certaines formes de "méthodes croisées" qui émergent dans le travail commun?

Considérer les pratiques d'enseignement et d'apprentissage comme des "faits sociaux totaux » qui, en tant que tels, contribuent à l'édification d'une culture, sans oublier le fait que l'École est un espace fortement ritualisé et que l'amélioration des conditions d'accès aux savoirs par tous sont dépendantes des nouveaux enjeux sociétaux, c'est considérer que les conditions d'existence des approches didactiques au sein des sciences sociales méritent un examen attentif et documenté.

Afin de contribuer à cet examen, deux axes étaient proposées aux intervenants - épistémologiques et opératoires - pour caractériser les relations entre didactique(s) et disciplines connexes dans les SHS. Les cas et points de vue ainsi présentés peuvent servir de points de départ à un débat que nous livrons ici sur les rapports entre approches didactiques de l'enseignement-apprentissage et l'étude des cultures et des formes d'activités humaines. D'une façon générale, l'objectivation de ces rapports nous semble être un enjeu majeur pour l'avenir des recherches en didactique, assumant leurs spécificités au sein des sciences de l'homme et de la société.

\section{CONTRIBUTIONS}

Les différentes contributions à cette thématique ont été organisées selon deux parties. Une première partie réunit les contributions de trois didacticiennes : Maryline Coquidé, Ecaterina BuléaBronckart et Marie-Pierre Chopin. Leurs propositions sont discutées par Chantal Amade-Escot. Une seconde partie réunit les contributions d'un philosophe de l'éducation (Henri Go) et d'un sociologue (Philippe Losego) et la discussion est conduite par Marie Toullec-Théry, didacticienne. Enfin, nous avons demandé à deux chercheures œuvrant dans des champs connexes à celui de la didactique (sciences de l'éducation pour Nathalie Younes et sociologie pour Marianne Woolven) de bien vouloir produire un regard distancié sur les débats qui ont animé cette journée de séminaire, conjointement organisée par l'Association pour des Recherches Comparatistes en
Didactique et la revue Éducation \& Didactique. À ce propos, nous tenons à signaler que les vidéos des différentes présentations sont en ligne ${ }^{9}$ sur le site de l'ARCD et accessibles aux adhérent(e)s.

\section{Maryline Coquidé, Stef Cachan, IFÉ et ENS de Lyon : Éléments rétrospectifs et perspectives pour la didactique des sciences et la didactique du curriculum}

Ce texte prend appui sur des travaux de recherche, individuels ou collectifs et concerne le domaine de la didactique des sciences et de l'approche curriculaire. Une étude historique de l'émergence de la didactique des sciences suivie de l'examen des relations entre didactique (des sciences et du curriculum) et d'autres domaines des sciences de l'homme et de la société (psychologie, sociologie, linguistique et histoire) montre que les interactions entre didactique et certaines SHS (en particulier anthropologie, phénoménologie et éducation comparée) sont encore insuffisantes.

M. Coquidé met en lumière le fait qu'à ses commencements, "la" didactique des sciences s'est nourrie d'un triple constructivisme (cognition, avec Piaget ; conceptualisation, avec Bachelard ; appropriation, avec Vygotski), qui nourrissent trois axes pour les recherches: constructivisme épistémologique, avec le concept central d'obstacle épistémologique ; constructivisme psychologique, avec celui d'obstacle psychogénétique; constructivisme pédagogique, avec celui d'obstacle didactique. Ces orientations ne prennent cependant pas en compte la perspective sociale. À la fin des années quatre-vingt-dix, Martinand, avec le concept de «pratique sociale de référence », contribuera à introduire l'éducation comme un fait social et conduira à envisager une approche curriculaire et non pas seulement disciplinaire des contextes scolaires et non scolaires. Par la suite, les évolutions et les élargissements du domaine de la recherche nécessitent d'autres regards centrés sur les acteurs et / ou les interactions et / ou sur les institutions. D'autres interfaces voient le jour : avec l'histoire et la sociologie du curriculum (Lebeaume); avec les sciences du langage, avec les travaux sur la place et le rôle du langage dans les interactions didactiques observées en situations naturelles (Bernié, Filliettaz \& Bronckart) ; avec les sciences du travail (ergonomie, psychologie du travail, sociologie des 
professions, recherches sur la professionnalité, didactique professionnelle...). On parle alors d'interfaces « historico-didactique » ou « socio-didactique ». Mais M. Coquidé s'interroge sur la réalité des champs de recherche ainsi esquissés : « que peuvent signifier ces termes juxtaposés, avec ou sans tirets, attribuées à ces nouvelles perspectives? Une articulation? Une reconfiguration? Une coordination des perspectives? Est-ce au niveau de la problématisation des recherches ? De l'analyse du corpus ? Comment exploiter une vraie richesse de l'altérité au sein des SHS, favoriser les échanges sur une question, tout en restant vigilant face à un risque de pulvérisation ou de dilution de l'objet de recherche? ». Sont alors discutées les exigences ou les modalités de mise en œuvre de ces relations au sein des SHS :

- par la prise en considération et donc le développement de certains « angles morts» : l'anthropologie « par rapport à la construction du sens pour le sujet, sans oublier la question de la langue »; la phénoménologie, portant «l'attention aux dimensions les plus corporelles et sensuelles de l'action »; l'éducation comparée, susceptible de dénaturer certains allant-de-soi des didactiques ;

- par le souci de développer de vraies « recherches partenariales », fondées à la fois sur une complémentarité et une confrontation, même si ces recherches " posent [...] de redoutables problèmes institutionnels et épistémologiques».

Ecaterina Bulea Bronckart, GRAFE, FPSE, université de Genève : Théories du langage et didactique de la grammaire : réflexions autour d'un dialogue suivi

Répondant à la demande d'illustrer certains aspects emblématiques du dialogue entre la recherche en didactique et les sciences humaines, E. Bulea-Bronckart traite ici d'un domaine volontairement étroit : celui des relations entre une « didactique de la grammaire » et les sciences du langage. Ce faisant, l'auteure fait le choix d'une certaine définition de la didactique, dont certains peuvent penser qu'elle est réductrice, mais qui est assumée ici comme étude de situations formelles au sein d'institutions qui font interagir des enseignants et des élèves pour que ceux-ci acquièrent des contenus définis par des prescriptions. Ceci posé, l'article
d'E. Bulea-Bronckart illustre la complexité des dynamiques d'échange entre un domaine des "sciences de l'enseignement" et les disciplines dites "de référence" : l'histoire de l'enseignement de la grammaire montre que la conception d'une circulation à sens unique, d'une transposition didactique simple, ne rend pas compte des faits. L'intérêt de sa contribution est d'illustrer ces phénomènes complexes, sur la longue durée, à partir de quelques exemples emblématiques : l'histoire de la notion d'adjectif, son usage dans l'enseignement et son traitement dans la théorie linguistique ; la Grammaire de Port-Royal, qui préfigure de manière frappante ce qui sera au $\mathrm{XX}^{\mathrm{e}}$ siècle le dialogue entre sciences du langage, sciences de la cognition et ingénierie; le modèle des formes et types de phrase, objet d'enseignement dont l'évidence masque mal qu'il est un construit théorique fragile. Ce que montre l'auteure, c'est que ces phénomènes ne relèvent donc pas tous d'une recherche de rationalité, mais reflètent des options plus politiques que scientifiques, qui viennent expliquer les contradictions et les errements dans la construction d'une didactique toujours écartelée par sa double nature d'ingénierie et de science. Comprendre certaines de ces difficultés apparemment "internes" au champ didactique est ainsi l'apport décisif d'une histoire et d'une sociologie de la science didactique, articulées à une épistémologie.

Comme le dit E. Bulea-Bronckart : " notre objectif est d'illustrer, par quelques épisodes qui nous paraissent parlants, l'existence d'un dialogue épistémique et disciplinaire autour du langage et de ses structures, afin d'en éclairer les développements actuels et d'alimenter la réflexion sur la direction dans laquelle ce même dialogue pourrait ou devrait se poursuivre».

Marie-Pierre Chopin, LaCES, université de Bordeaux : Anthropologie de la diffusion des savoirs : genèse et évolution de l'approche anthropo-didactique des phénomènes d'éducation

Dans cette contribution, M.-P. Chopin procède à l'analyse de la genèse et de l'évolution de l'approche dite " anthropo-didactique » (AD) des phénomènes d'éducation, développée depuis une vingtaine d'année sous l'impulsion de B. Sarrazy au sein d'une équipe de recherche bordelaise en sciences de l'éducation. Située à l'origine, comme son nom l'indique, 
au croisement de regards anthropologiques et didactiques, l'approche tend aujourd'hui à se présenter plus directement comme une " anthropologie de la diffusion des savoirs ». Il s'agit de montrer que cette évolution n'est pas sans lien avec la question au centre du séminaire.

Dans cette approche, « la didactique a été conçue d'emblée comme une anthropologie », et de fait, dès le début la Théorie des Situations Didactiques (TSD) de Brousseau sera reconsidérée sous différents points de vue : celui de la psychologie piagétienne, celui des anthropologies de l'usage (Wittgenstein), celui du langage (Searle) et encore celui de la pratique (Bourdieu), sans négliger pour autant ceux de l'anthropologie post-culturaliste américaine (Geertz, Mc Dermott ou Ogbu). Forte de cet arrière-plan épistémologique, l'AD se propose de développer en tant que telle la dimension implicitement anthropologique de la TSD, aussi bien en termes méthodologiques ( son rapport au terrain de la recherche et, plus fondamentalement, à l'empirie et à la contingence ») qu'en termes de focus («sa circonscription de l'objet de la didactique, non focalisé sur les personnes, mais sur les positions qu'elles occupent au sein des espaces de pratiques scolaires et sur les relations qui les unissent à leur milieu »). En effet, le questionnement typiquement ethnographique permet en particulier de questionner les conditions mêmes de production des données de recherche dans l'observation. Quant au choix de l'objet relation, il permet, selon M.-P. Chopin, de s'opposer tant au psychologisme qu'au sociologisme, tout en respectant un principe éthique qui affirme que le sens des actions appartient d'abord aux acteurs, en référence à Mc Dermott. L'auteure fait ainsi la démonstration du potentiel heuristique de l'AD que ce soit au niveau du développement de concepts majeurs de la Théorie des Situations (institutionnalisation, contrat didactique, dévolution), que de l'étude des sociétés éducatives du point de vue de l'épaisseur et de la dynamique des situations (arrière-plans, idéologies, mémoires, temps de la diffusion des savoirs...). Ce dialogue anthropologico-didactique focalisé sur l'étude des situations a permis l'émergence de nouveaux concepts qui permettent, entre autre, de renouveler l'intelligibilité de certains phénomènes d'enseignement et d'apprentissage liés à la difficulté scolaire. Ce qui se dessine, selon M.-P. Chopin, c'est que dans son projet fondamental, toute didactique est nécessairement anthropologie.

\section{Discussion - Chantal Amade-Escot, EFTS, université de Toulouse Jean-Jaurès}

Nous attirons l'attention du lecteur sur l'aspect singulier de ce texte qui correspond à la transcription remaniée de la réaction de C. Amade-Escot à ces trois premières contributions.

La discussion menée par l'auteure prend en compte successivement les deux axes pointés dans le cadrage de la journée : l'axe épistémologique et l'axe opératoire qui viennent tous deux interroger l'ambition de penser les / la didactique(s) comme composante des sciences de l'homme et de la société. En ce qui concerne l'axe épistémologique, les trois textes interpellent le dialogue entre la didactique et l'anthropologie comme une affirmation d'un éloignement entre didactique et psychologie : le cœur de la préoccupation des conférencières relève des situations et non pas du sujet. Se pose alors la question de la légitimation des didactiques et des réticences. En effet, si les didacticiens dans leurs travaux interrogent régulièrement les emprunts et les conversions de concepts issus des SHS, en retour, on ne sait que peu de choses de la reprise par les autres disciplines des textes fondamentaux du champs de la didactique. Une autre manière de percevoir la légitimation est d'observer la manière dont les didacticiens obtiennent des contrats sur appels à projets scientifiques : il apparaît nécessaire de s'appuyer sur des sociologues, des psychologues... En terme de réticence, il faut également admettre qu'il est bien difficile à la recherche didactique de faire reconnaître ses résultats au sein du système institutionnel. Pour autant, les problématiques traitées par les didactiques sont largement partagées avec d'autres sciences sociales et suggèrent donc des collaborations, ne serait-ce que comme moyen de validation des résultats et des modèles théoriques par leur confrontation raisonnée et critique. Cet axe épistémologique interroge dans les trois textes la nécessité d'historiciser les relations entre les didactiques naissantes et leurs emprunts dans les SHS afin de porter la réflexion sur les nécessités contextuelles qui permettent de mieux saisir l'évolution des rapports. Se pose alors la question d'un élargissement des collaborations qui, dans l'ignorance des arrière-plans, peuvent développer un universalisme réducteur ou une interdisciplinarité fourre-tout. Malgré tout, on ne peut parler du rapport des didactiques au champ des SHS car ce rapport est nécessairement divers, que certaines didactiques 
parlent de disciplines contributives ou au contraire que d'autres revendiquent un rapport plus autonome.

C'est là que l'approche comparatiste intervient en produisant, dans ses travaux, un déplacement de perspective : d'une perspective épistémologique, vers une perspective plus pragmatiste qui prend en compte l'agentivité des acteurs.

Concernant l'axe opératoire de la question des relations didactique(s)-SHS, C. Amade-Escot, en écho aux trois contributions interroge ce qui peut être partagé : des questions, des élaborations de problématique, des objets d'étude communs, des enjeux et des visées, des constructions méthodologiques originales? Le texte de cadrage propose une définition du didacticien comme un ingénieur et un chercheur. Même si l'auteure y souscrit, elle ne peut que relever la tension qui s'en suit entre dimension épistémique et transformative et la difficulté à situer les avancées des travaux menés en didactique dans les dernières réformes. Une explication est la tendance des systèmes éducatifs à penser la question de l'enseignement et de l'apprentissage selon un modèle descendant, indépendamment des acteurs et de leur agentivité.

Enfin, cette discussion se termine sur une invitation à penser des objets frontières dans le cadre de courtes problématisations conjointes.

\section{Philippe Losego, HEP Vaud : Le social et le didactique : distance et rapprochement}

Ce texte examine le mouvement qui rapproche sociologues et didacticiens depuis le début des années deux-mille. Aujourd'hui si la sociologie peut discuter des programmes ou des curricula de manière à la fois relativiste et respectueuse des savoirs et que les didactiques se rapprochent de plus en plus de la sociologie et de l'anthropologie en général, il faut cependant envisager les malentendus et les difficultés. Pour cela, le sociologue P. Losego commence par faire l'histoire de sa propre discipline, en regard de sa position sur la question spécifique des savoirs. En effet, pourquoi la sociologie elle-même s'est souvent désintéressée des savoirs, notamment des savoirs scolaires ? Le problème posé est celui de la possibilité d'envisager le caractère socialement construit des savoirs sans pour autant en faire de purs arbitraires. Il reprend, depuis Weber, une synthèse des positions des « critiques de l'arbitraire culturel d'inspiration à la fois marxiste et wébérienne » (Verret, Bourdieu \& Passeron), qui dénonce le coup de force par lequel « l'école impose une seule culture, qu'elle présente comme universelle alors qu'elle relève d'un arbitraire de classe ». Selon P. Losego, ce courant de la sociologie critique est à distinguer de la «théorie marxiste de la mauvaise conscience " représenté par Baudelot et Establet puis Tanguy, qui « ne remettent pas en question la culture humaniste, bien au contraire » mais « déplorent qu'elle soit de fait interdite aux enfants de la classe populaire ». Les travaux qui suivent à partir des années quatre-vingtdix, dénotent un changement de focale et remettent au premier plan les savoirs, s'interrogeant sur la formation des programmes et la possibilité de définir des « socles communs» légitimes, tout autant que sur les origines sociales des difficultés des élèves, à l'échelle des pratiques scolaires saisies à une échelle fine (Charlot, Bautier \& Rochex ; Lahire ; Rochex \& Crinon) : désormais, la sociologie des apprentissages se rapproche ainsi de la perspective didactique. Les sociologues commencent à s'intéresser à la construction des programmes scolaires (Baluteau, 1999) comme des « choses » en soi et non pas seulement comme support de validation de théories sociologiques globale (reproduction, distribution sociale des savoirs, etc.).

En regard, il est difficile de juger d'un champ unifié du côté de la didactique. P. Losego choisit de contraster, du point de vue de leur rapport aux problématiques sociologiques, la didactique des mathématiques et la didactique du français. Les didactiques semblent se caractériser par la tendance au réductionnisme, considérant un Élève, une Classe, le Savoir... comme des abstractions sans dimension sociale. Mais la didactique du français, lectrice de Bernstein et de Lahire, va plus tôt que la didactique des mathématiques, réintroduire un questionnement sociologique dans ses approches, d'abord en s'intéressant aux situations "naturelles" d'enseignement et aux méthodes ethnologiques, ensuite en considérant les élèves comme des acteurs dont on peut suivre les conceptions et l'action à plusieurs échelles. Au cours des années deux-mille, les didactiques vont traiter enseignants et élèves comme des acteurs sous contraintes dans des systèmes (didactiques), ce qui pourrait ressembler à une définition venue de la sociologie.

Reste que les relations entre sociologie et didactique sont encore plus de contact que de collabora- 
tion, ce qui amène l'auteur à considérer les compatibilités autant que les malentendus conceptuels. En effet, " si les didactiques affirment aujourd'hui prendre en compte le "social", c'est parfois sur la base de notions contre lesquelles une bonne partie de la sociologie s'est construite, comme la "demande sociale" ou les "finalités sociales" qui sous-entendent souvent une cohérence sociétale, alors que la plupart des sociologues considèrent la société comme un ensemble de contradictions et de contingences $»$. De même, le " collectif » en didactique semble monolithique et agir littéralement " comme un seul élève ", hypothéquant ainsi l'étude de sa dimension dynamique et problématique.

P. Losego conclut que "s'il n'y a pas de frontière entre forme et contenu, entre le "social" et le "scolaire", il ne pourrait y avoir de frontière entre sociologie et didactique ».

Henri-Louis Go, LISEC, université de Lorraine : La philosophie a-t-elle quelque chose à dire à la didactique?

De nombreux philosophes se sont exprimés sur la transmission de normes, de savoirs, d'une culture, il est donc habituel de philosopher à propos de questions d'éducation, et l'on peut même convenir que « certains des problèmes cruciaux de l'institution scolaire d'aujourd'hui sont pour une part réductible à des problèmes philosophiques » (Kambouchner, 2013, p. 9). H.-L. Go assume d'emblée le fait que l'on peut faire un travail philosophique concernant différents problèmes relatifs à l'éducation.

La contribution de cet auteur illustre par sa démarche même l'intérêt d'un échange entre approche philosophique et approche didactique du phénomène éducatif : au lieu de s'attaquer directement au(x) concept(s) de didactique(s), H.-L. Go déplace le problème pour l'aborder par ses fondements. Il commence par rappeler ce qu'est le travail philosophique par rapport au projet d'élaboration de connaissance ou de conception d'intervention que vise la recherche en didactique. Que font les philosophes ? "Quand on réfléchit en philosophie, c'est que l'on "a" un problème, que l'on éprouve une difficulté. Étymologiquement, ce que l'on appelle problème c'est ce qui fait obstacle ». L'auteur montre ensuite comment la philosophie permet d'interroger le sens que l'on veut donner à l'éducation dans la problématicité du monde actuel. Plus qu'une manière de figer des réponses, l'acte de philosopher réside dans la capacité à (re)poser les problèmes, à approfondir ce que signalent les obstacles et à maintenir vive la capacité d'étonnement au sein d'un « monde problématique ». Ainsi, "l'éducation ne peut consister seulement à conformer les nouveaux venus à des normes pré-données, mais éduquer engage l'éducateur à assumer un discours sur le monde, et à assumer des formes d'action éducative ». Ces formes relèvent du devoir de transmission de normes culturelles et éthiques mais ces dernières poursuivent des finalités contrastées : préparer l'enfant à vivre dans un monde qui lui préexiste mais en même temps l'amener à questionner le monde et à rencontrer des œuvres, pour réinventer ce monde. L'auteur en vient alors à examiner de plus près ce que signifie être professeur. En distinguant le sens que peut avoir l'acte même de professer, qu'il distingue d'enseigner, de transmettre ou d'instruire, il pose la question de ce qu'est instruire : s'agit-il de transmettre un savoir formel et figé sur le monde, ou s'agit-il de favoriser (pour les élèves) une expérience du monde, et un pouvoir pratique dans le monde? «Le monde récité par le professeur conformiste n'est qu'une fiction scolaire. » Cela conduit alors H.-L. Go à prendre au sérieux ce qu'il appelle volontiers l'interrogation sceptique du métier de professeur, voire la thérapie sceptique en vue de mieux cerner de quelle responsabilité le professeur est en charge. En effet, l'auteur nous convie à examiner l'aporie suivante : "la forme scolaire républicaine demande au professeur la neutralité face au monde; c'est la rigueur laiqque. Cependant, la forme scolaire démocratique a besoin de la vigueur intellectuelle du professeur qui sait exercer son jugement critique dans le métier et sur le monde ». Dans ce texte, H.-L. Go n'apporte pas seulement une méthode intellectuelle fondée sur un scepticisme méthodique à l'égard de ce que présuppose une formation d'enseignant : il en rappelle la dimension essentiellement éthique et politique.

\section{Discussion - Marie Toullec Théry, CREN, université de Nantes: Voir la scène scolaire comme un contexte pluriel, selon diverses échelles}

Cette contribution est une tentative de faire converser deux points de vue, celui d'un philosophe didacticien, ancré dans la Théorie de l'Action 
Conjointe en Didactique - Henri-Louis Go - et celui d'un sociologue - Philippe Losego qui interroge le caractère « réductionniste » des approches didactiques. L'enjeu de cette discussion est de déceler des objets plus transversaux qui permettraient d'envisager des espaces communs de problématisation, au sens de Rayou (2014). Une première partie, en appui sur des travaux de didacticiens et de sociologues, tente d'introduire d'autres questions et de circonscrire certains obstacles majeurs de ce projet social au sein des sciences de l'homme et de la société. L'auteure pose alors une question sous forme de provocation : les métiers de la didactique valent-ils d'être étudiés ? Au sein des sciences de l'homme et de la société, la didactique et le didactique seraient donc des objets à la fois triviaux et trop liés à la sphère individuelle des enseignants pour être étudiés. L'auteure choisit donc d'investir ce qui ressort de ce rapport entre collectif et individuel dans les textes de Go et Losego.

Elle montre que d'une méfiance vis-à-vis des savoirs en tant qu'objet transparent, on assiste actuellement à un changement notoire qui s'opère actuellement puisque d'un mouvement premier de non pertinence de la prise en compte des savoirs émerge la nécessité de l'étude de ces savoirs, entre autre parce qu'ils créent des inégalités. De fait, la scène scolaire vaudrait-elle alors d'être étudiée? M. Toullec-Théry en vient alors à identifier quelques nécessités inhérentes à la nature même de cette scène scolaire. Tout d'abord, celle de voir cette scène comme une scène politique mue par des savoirs, et qui se caractériserait par une triple dimension qui relève à la fois de l'éthique, de l'héritage et de la production d'objets propices à une émancipation de l'individu via des savoirs. Ensuite, cette scène scolaire, en tant qu'observatoire anthropologique de la pratique du métier, peut être vue comme celle qui érige l'expérience en phénomène. Une troisième nécessité tient au contexte spécifique et générique de cette scène scolaire qui suppose des échelles d'analyse différentes pour prendre ensemble les contraintes du statut de l'élève dans ses dimensions cognitives, culturelles et collectives et accéder plus finement aux déterminations de l'action des enseignants (Toullec-Théry \& Marlot, 2013). Le détour par ces diverses nécessités propres à la scène scolaire, montre que celleci vaut donc d'être étudiée parce que l'expérience y est spécifique et sujette à diverses influences (institutionnelles, curriculaires, locales...). C'est là un appel explicite à la nécessité du dialogue au sein des sciences sociales.

L'auteure conclut sa discussion par l'identification de trois notions candidates à ce rapprochement entre sociologie et didactique : le contrat didactique, les malentendus socio-cognitifs et l'épistémologie pratique du professeur. Ce rapprochement prend forme au travers de l'approche comparatiste en didactique. La discussion se clôt sur un point de désaccord entre les deux auteurs, Go et Losego et renvoie à la dialectique individuel-collectif. Si pour le premier, le collectif en didactique lui semble monolithique, pour le second, il s'agit de comprendre de quelles manières professeur et élèves s'approprient des usages collectifs construits.

\section{Marianne Woolven \& Nathalie Younes, ACTÉ, université Blaise-Pascal, Clermont-Ferrand : Entre disciplines.}

La contribution qui vient clore ce numéro cerne à la fois les éléments saillants relatifs à la question du dialogue entre SHS et didactique(s) mais tente également de mettre au jour certaines zones d'ombres et d'impensés que pourrait révéler l'ensemble des textes. Trois axes de questionnement sont ainsi proposés par les deux auteures : le rattachement des didactiques aux SHS, le paradoxe des liaisons et déliaisons de la didactique avec les SHS et la question de la collaboration.

Si le rattachement de la didactique aux SHS semble aller de soi pour les différents auteurs de ce numéro, un regard historique sur les sciences humaines et sociales permet de constater que la question des échanges, des transferts mais aussi des frontières se pose de manière récurrente depuis le $\mathrm{XIX}^{\mathrm{e}}$ siècle et que ce questionnement autour d'un dialogue possible s'inscrit bien dans les débats classiques des SHS, qu'il s'agisse de ceux propres aux sociologues, comme aux psychologues.

Le deuxième axe vient interroger la nature des liens entre la didactique, les sciences de l'éducation et la psychologie d'une part et l'anthropologie d'autre part. Les références citées par les différents auteurs tendent à montrer que dans les domaines des sciences de l'éducation et de la psychologie, on assiste à un déplacement des référents prioritaires de la psychologie à la sociologie. En revanche, les auteurs montrent que dans les publications les plus 
récentes (Plaisance, 2012 ; Laot \& Rogers, 2015), la didactique est à peine évoquée. Pour ce qui est de l'anthropologie, les auteurs mettent en lumière que sous un apparent consensus et des acceptions proches, diverses conceptions sont en fait à l'œuvre. Ces spécifications conduisent alors à des formes de dialogue nécessairement distinctes mais sans que les interlocuteurs et les modalités d'échange soient identifiés.

Dans un premier sens (Go, Coquidé), l'anthropologie constitue un apport de connaissances pour l'étude des phénomènes éducatifs mais sans que soit précisé comment le travail des anthropologues pourrait être réapproprié par les didacticiens. Un bon exemple de réappropriation semble être le cas de l'ouvrage récent de $\mathrm{M}$. Laparra et $\mathrm{C}$. Margolinas (2016).

Dans un second sens, il s'agirait non pas tant d'une discipline des SHS que d'un aspect de la réalité. Dans ce numéro, ce ne sont donc pas les problématiques de l'anthropologie telles que l'enquête de terrain, ou l'altérité qui sont considérées.

Les deux auteures montrent que le troisième sens, celui de « l'anthropologique » comme ce qui est propre à l'humain, porté par des chercheurs comme Chevallard (2014) ou encore Sensevy (2009) et repris par plusieurs auteurs (Chopin, Toullec-Thery) témoigne en fait d'une volonté de montée en généralité de la part des didacticiens. Dans le même temps, cette volonté épingle la pertinence du dialogue souhaité par ces derniers.

Le troisième axe vient interroger la dimension opératoire du dialogue avec la question des collaborations. À ce propos, même si les auteures ne peuvent que constater que les didactiques et les sciences de l'éducation se sont construites en parallèle, dans une relative ignorance, elles mettent la focale sur deux chantiers, potentiellement féconds : celui de l'évaluation avec la création du réseau EVADIDA (ÉVAluation et DIDActique) construit au sein de l'ADMEE (Association pour le Développement des Méthodologies d'Évaluation en Éducation) et le chantier épistémologique propre à articuler les relations entre niveaux d'explication pour rendre compte de manière complète d'une situation donnée.

En conclusion, si de leur point de vue, les deux auteures saluent la volonté des auteurs du numéro d'identifier les acculturations réciproques et leurs perspectives heuristiques, elles révèlent certains impensés qui tiennent à la conception de l'expéri- mental ou encore à l'opacité des champs lexicaux qui génèrent des malentendus. Elles terminent par un renversement des propositions : et si les autres champs disciplinaires des SHS étudiaient maintenant la réception qu'ils font des questions et notions issues de la didactique?

\section{NOTES}

1. [https://www.cairn.info/revue-education-et-didactique-2014-1.htm]

2. [http://www.arcd.fr/lassociation/l

3. [http://educationdidactique.revues.org/]

4. [http://ife.ens-lyon.fr/publications/edition-electronique/ education-societes/RE004-3.pdf]

5. [http://educationdidactique.revues.org/86]

6. [http://www.hepl.ch/files/live/sites/systemsite/files/ueragirs/actualites/colloque-didactiques-et-sociologie/actescolloque-sociologie-et-didactiques-philippe-losego-ueragirs-hep-vaud.pdf]

7. [http://rfp.revues.org/4522]

8. [http://educationdidactique.revues.org/1847]

9. [http://www.arcd.fr/activites-scientifiques/ journees-detude/journee-arcd-ed-2015/] 


\section{RÉFÉRENCES}

Baudelot, C., \& Establet, R. (1971). L'école capitaliste en France. Paris : Maspéro.

Bernié, J. P. (2002). Lapproche des pratiques langagières scolaires à travers la notion de « communauté discursive ». Un apport à la didactique comparée ? Revue Française de Pédagogie, (141), 77-88.

Bourdieu, P., \& Passeron, J.-C. (1970). La Reproduction. Éléments pour une théorie du système d'enseignement. Paris : Minuit.

Bronckart, J. P. (1987). Les sciences du langage, un défi pour l'enseignement? Neuchâtel : Delachaux \& Niestlé.

Charlot, B., Bautier, E., \& Rochex, J.-Y. (1992). École et savoir dans les banlieues... et ailleurs. Paris : Armand Colin.

Chevallard, Y. (2014). Des didactiques des disciplines scolaires à la didactique comme science anthropologique. Sur un obstacle épistémologique, psychologique et institutionnel. Éducation \& Didactique, 8(1), 35-44.

Daunay, B., Fluckiger, C., \& Hassan, R. (dir.) (2015). Les contenus d'enseignement et d'apprentissage. Approches didactiques. Bordeaux : Presses universitaires de Bordeaux.

Éducation \& Didactique, 8(1). (2014). Didactiques et / ou didactique? D'une question polémique à la construction d'un espace de problématisation scientifique (Actes de la journée ARCD / Éducation \& Didactique). Rennes : Presses universitaires de Rennes.

Filliettaz, L., \& Bronckart, J.-P. (dir.). (2005). Lanalyse des actions et des discours en situation de travail. Concepts, méthodes et applications. Louvain-la-Neuve : Peeters.

Johsua, S., \& Lahire, B. (1999). Pour une didactique sociologique. Entretien avec Samuel Johsua, professeur à l'université d'Aix-Marseille, didacticien des sciences et des mathématiques. Éducation E Société, 2(4), 29-56.

Kambouchner, D. (2013). L'école, question philosophique. Paris : Fayard.

Lahire, B. (2007). La sociologie, la didactique et leurs domaines scientifiques. Education $\&$ didactique, 1(1), 73-81.
Laot, F., \& Rogers, R. (dir.) (2015). Les sciences de l'éducation. Émergence d'un champ de recherche dans l'aprèsguerre. Rennes: Presses universitaires de Rennes.

Laparra, M., \& Margolinas, C. (2016). Les premiers apprentissages scolaires à la loupe. Des liens entre énumération, oralité et littératie. Louvain-la-Neuve : De Boeck.

Ligozat, F., Coquidé, M., Marlot, C., Verscheure, I., \& Sensevy, G. (2014). Didactiques et / ou didactique. Poursuivre le travail de problématisation. Éducation $\mathcal{E}$ Didactique, 8(7), 101-115.

Losego, P. (dir.) (2014a). Sociologie et didactiques : traverser les frontières. Revue française de pédagogie, (188).

Losego, P. (dir.). (2014b). Actes du colloque "Sociologie et didactiques ": vers une transgression des frontières, 13 et 14 septembre 2012. Lausanne : Haute École Pédagogique de Vaud. Repéré à [http://www.hepl.ch/ sociodidac]

Mercier, A, Sensevy, G., \& Schubauer-Leoni, M. (2002). Vers une didactique comparée. Revue française de pédagogie, (141), 5-16.

Plaisance, E. (2012). Les sciences de l'éducation. Paris : La Découverte.

Rayou, P. (2014). Sociologie et didactique. Vers un espace commun de problématisation. Éducation \& Didactique, 8, 91-99.

Rochex, J.-Y., \& Crinon, J. (2011). La construction des inégalités scolaires au coeur des pratiques et dispositifs d'enseignement. Rennes: Presses universitaires de Rennes.

Sensevy, G. (2009). Didactique et sciences de l'éducation : une reconfiguration? Dans A. Vergnioux (dir.), 40 ans des sciences de l'éducation (p. 49-58). Caen : Presses universitaires de Caen.

Toullec-Théry, M., \& Marlot, C. (2013). Les déterminations du phénomène de différenciation didactique passive dans les pratiques d'aide ordinaire à l'école élémentaire. Revue française de pédagogie, (182), 41-53.

Verret, M. (1975). Le temps des études. Paris : Honoré Champion. 